Review Article

\title{
COVID-19: Gastrointestinal Manifestations and Complications
}

\author{
Angel Yun-Kuan Thye ${ }^{1}$, Priyia Pusparajah ${ }^{1}$, Loh Teng-Hern Tan ${ }^{1,2}$, Jodi Woan-Fei \\ Law $^{1}$, Vengadesh Letchumanan ${ }^{1 *}$, Learn-Han Lee ${ }^{1 *}$ \\ Article History \\ Received: 15 October 2021 ; \\ Received in Revised Form: \\ 14 November 2021; \\ Accepted: 15 November \\ 2021; \\ Available Online: 22 \\ November 2021

\begin{abstract}
${ }^{1}$ Novel Bacteria and Drug Discovery (NBDD) Research Group, Microbiome and Bioresource Research Strength (MBRS), Jeffrey Cheah School of Medicine and Health Sciences, Monash University Malaysia, Bandar Sunway, Subang Jaya, Selangor, 47500, Malaysia; angelthye.yunkuan@monash.edu (AY-KT); priyia.pusparajah@monash.edu (PP); jodi.law1@ monash.edu (JWFL)

${ }^{2}$ Clinical School Johor Bahru, Jeffrey Cheah School of Medicine and Health Sciences, Monash University Malaysia, Johor Bahru 80100, Malaysia; loh.teng.hern@monash.edu (LT-HT)

*Corresponding author: Vengadesh Letchumanan, Learn-Han Lee; Novel Bacteria and Drug Discovery (NBDD) Research Group, Microbiome and Bioresource Research Strength (MBRS), Jeffrey Cheah School of Medicine and Health Sciences, Monash University Malaysia, Bandar Sunway, Subang Jaya, Selangor, 47500, Malaysia; vengadesh.letchumanan1@ monash.edu (VL), lee.learn.han@monash.edu (L-HL)
\end{abstract}

\begin{abstract}
The virus responsible for the COVID-19 pandemic is the severe acute respiratory syndrome coronavirus 2 (SARS-CoV-2), which belongs to the genus Betacoronavirus. This genus also includes the severe acute respiratory syndrome coronavirus (SARS-CoV) and the Middle East respiratory syndrome coronavirus (MERS-CoV). The common symptoms of COVID-19 infection are fever and respiratory symptoms, but it can also involve the gastrointestinal tract (GIT), resulting in manifestations such as diarrhea, nausea and/or vomiting and abdominal pain. The emergence of COVID-19 led to public health emphasis on droplet transmission and precautions of contact with respiratory secretions. However, mounting evidence demonstrates detection of SARS-CoV-2 RNA in stool samples of COVID-19 patients. It has also been demonstrated that the host receptor angiotensinconverting-enzyme-2 (ACE-2) is highly expressed not just in respiratory cells but also in gastrointestinal sites involving the glandular cells of gastric, duodenal, and rectal epithelium. This suggests that SARS-CoV-2 can infect the digestive system, serving as another route of transmission. This review aims to study the prevalence of some of the gastrointestinal manifestations following COVID-19 infection and findings of positive SARS-CoV-2 RNA in stool specimens while making parallels to the severe acute respiratory syndrome (SARS) and the Middle East respiratory syndrome (MERS) infection. We will also discuss the possible pathophysiology of COVID-19 related gastrointestinal involvement.
\end{abstract}

Keywords: COVID-19; SARS-CoV-2 RNA; gastrointestinal; angiotensin converting enzyme 2 (ACE-2); stool

\section{Introduction}

The Coronavirus Disease 2019 (COVID-19) was declared a pandemic by the World Health Organization (WHO) in early March $2020^{[1-3]}$. This global pandemic started with an 
outbreak of pneumonia of unknown aetiology in Wuhan, Hubei province of China ${ }^{[4-8]}$. The virus responsible for causing COVID-19 was named the severe acute respiratory syndrome coronavirus 2 (SARS-CoV-2) by the Coronavirus Study Group of the International Committee on Taxonomy of Viruses (ICTV) ${ }^{[9]}$. It is a positive sense, single-stranded RNA virus belonging to the genus Betacoronavirus that originated from bats ${ }^{[4,10]}$. Interestingly, SARS-CoV-2 has a 79\% overlap of genomic sequence identity with the severe acute respiratory syndrome coronavirus (SARS-CoV) and 50\% with the Middle East respiratory syndrome coronavirus (MERS-CoV) ${ }^{[11]}$. At the time this paper went to press, the SARSCoV-2 had infected over 250,000,000 people and caused 5,072,046 deaths worldwide (as of $10^{\text {th }}$ November 2021) ${ }^{[12]}$. The virus spread swiftly worldwide, with the emerging of new variants of concern (VOC) with a higher infectivity linked to high fatality rates ${ }^{[13]}$.

The data suggests that SARS-CoV-2 primarily infects respiratory epithelial cells and spreads via respiratory route from human to human, however, the exact viral target cells and organs are yet to be determined ${ }^{[14]}$. Some of the most common symptoms of COVID-19 infections include fever and respiratory symptoms such as dry cough and dyspnea, which is similar to the severe acute respiratory syndrome (SARS) in 2003 and Middle East respiratory syndrome (MERS) in 2012. Aside from respiratory symptoms, gastrointestinal symptoms including diarrhea, nausea, vomiting and abdominal pain may also be present ${ }^{[15]}$. There has been escalating evidence of SARS-CoV-2 RNA being detected in stool specimens ${ }^{[14,16,17]}$, and anal ${ }^{[18]}$, or rectal swabs ${ }^{[19]}$, of COVID-19 patients despite the clearance of SARS-CoV-2 in the respiratory cells/upper respiratory $\operatorname{tract}^{[14,18,20]}$. Besides that, the angiotensinconverting enzyme-2 (ACE-2) receptor is highly expressed in gastrointestinal epithelial cells, particularly the ileum, duodenum, jejunum, caecum and colon ${ }^{[14,21]}$. Taken together, these suggest that SARS-CoV-2 may also demonstrate fecal-oral transmission, in addition to droplet transmission; which has implications on SARS-CoV-2 transmission, infection control and management. The aim of this review is to study the prevalence of some of the gastrointestinal symptoms and the positive detection of SARS-CoV-2 RNA in stool specimens of COVID-19 patients, while making parallels to SARS and MERS infection. We will also discuss some of the possible causes of gastrointestinal involvement following COVID-19.

\section{Prevalence of Gastrointestinal Symptoms of COVID-19}

The well-established signs and symptoms of COVID-19 patients are fever and respiratory symptoms. However, gastrointestinal symptoms such as diarrhea, vomiting and abdominal pain seem to frequently also be part of the manifestations of the disease. In the United States, the first confirmed COVID-19 case was reported on the $20^{\text {th }}$ January 2020 
involving a 35-year-old man who just returned from Wuhan, China. Upon admission, he presented with persistent dry cough and a 2-day history of nausea and vomiting. On the second day of hospitalization, he reported diarrhea and abdominal discomfort ${ }^{[16]}$.

In a meta-analysis consisting of 4,243 COVID-19 positive patients (60 studies) from 6 countries (China $n=53$, South Korea and Singapore $n=2$, Vietnam, United States and United Kingdom $n=1$ ) the pooled prevalence of gastrointestinal symptoms was $17.6 \%{ }^{[22]}$. For individual gastrointestinal symptoms, 18 studies reported the prevalence as follows: lack of appetite (26.8\%), diarrhea (12.5\%), nausea/vomiting (10.2\%), and abdominal pain $(9.2 \%)^{[22]}$. A cross sectional multicenter study involving 204 hospitalized COVID-19 positive patients in China's Hubei province found 103 (50.5\%) patients had $\geq 1$ digestive symptoms, including diarrhea (34\%), vomiting (3.9\%), abdominal pain (1.9\%) ${ }^{[23]}$. Furthermore, in a Hong Kong cohort with 59 COVID-19 positive patients, 15 (25.4\%) patients presented gastrointestinal symptoms of diarrhea (22\%), abdominal pain (11.9\%), vomiting (1.7\%), and $9(15.3 \%)$ had positive viral RNA in stool specimens ${ }^{[22]}$. A few other cohorts also reported frequencies of diarrhea ranging $2.0-10.1 \%$ and nausea and/or vomiting ranging $1.0-10.1 \%^{[10,24-32]}$.

Similar to adults, children also displayed gastrointestinal symptoms following positive COVID-19 assays. However, in terms of COVID-19 severity, children and/or adolescents were found to have less severe COVID-19 infection, with milder symptoms and possibly better prognosis than adults ${ }^{[33,34]}$. In a meta-analysis of 280 COVID-19 positive children, the pooled prevalence of gastrointestinal symptom was $22.8 \%$. The pooled prevalence of diarrhea, vomiting and abdominal pain was respectively $12.4 \%, 10.3 \%$, and $5.4 \%$ respectively ${ }^{[35]}$. On the other hand, another meta-analysis consisting of 2855 children showed $4 \%$ had diarrhea and none had abdominal pain ${ }^{[33]}$. The variability in rates in different studies could be a result of variability in clinical presentation and study size. Hence, more clinical data is needed.

Similar to COVID-19, SARS and MERS outbreaks also demonstrated gastrointestinal (GI) manifestations during the course of the disease. A retrospective study in Hong Kong involving 138 SARS patients revealed diarrhea to be the most common GI symptom with an average $( \pm$ SD) duration of diarrhea of $3.7 \pm 2.7$ days. Out of 138 patients, 28 (20.3\%) had diarrhea at presentation while $53(38.4 \%)$ had diarrhea at the first 3 weeks of illness ${ }^{[36]}$. Using reverse transcriptase-polymerase chain reaction (RT-PCR), it was also demonstrated that the stool specimens from SARS patients had a $16 \%$ detection rate of severe acute respiratory syndrome coronavirus (SARS-CoV) RNA, comparable to the detection rate in nasopharyngeal aspirates ${ }^{[36]}$. MERS also presented with gastrointestinal symptom - in a study of 47 MERS infected patients in Saudi Arabia, diarrhea (26\%), vomiting (21\%) and 
abdominal pain (17\%) were present at presentation ${ }^{[37]}$. A retrospective observational study from Korea with 186 MERS patients also showed gastrointestinal symptoms of diarrhea $(19.4 \%)$, nausea and vomiting $(14 \%)$ and abdominal pain $(8.1 \%)^{[38]}$.

It is clear that COVID-19 has a similar gastrointestinal presentation as that seen in SARS and MERS. COVID-19 positive patients whether children or adults can present with gastrointestinal symptoms early in the course and it should not be taken lightly. For instance, a study found nearly a quarter of COVID-19 positive children have at least one gastrointestinal symptom with diarrhea being the most common symptom followed by vomiting and abdominal pain ${ }^{[35]}$. This finding was quite consistent with a meta-analysis in adult COVID-19 patients that found the prevalence of gastrointestinal symptoms to be $18 \%{ }^{[22,35]}$. Nonetheless, there could be variation in the rate of prevalence among different studies due to variability in study size and clinical presentation.

\section{Detection of SARS-Cov-2 RNA in Stool Specimens of COVID-19 Patients}

In relation to SARS-CoV-2 RNA detection in stool specimens, US's first COVID-19 positive patient presented with gastrointestinal symptoms and his stool sample together with respiratory specimens (nasopharyngeal and oropharyngeal) and serum were sent for real time RT-PCR testing in which both stool (illness day 7) and respiratory specimens (illness day 4 and day 7) detected SARS-CoV-2 RNA ${ }^{[16]}$. It is also important to note that there is also positive live SARS-CoV-2 detection in stool samples of patients without diarrhea ${ }^{[17]}$. A study showed among children tested for SARS-CoV-2 by stool PCR or rectal swab, $89 \%$ had positive results even though $82.6 \%$ of them had no gastrointestinal symptoms ${ }^{[19]}$. This finding is consistent with a Hong Kong cohort where 15.3\% of COVID-19 patients have SARS-CoV2 RNA detected in their stool specimen on presentation regardless of whether or not they have gastrointestinal symptoms, and when compared to those without diarrhea, those with diarrhea have higher stool RNA positivity and viral load ${ }^{[22]}$. This suggests, with or without gastrointestinal symptoms, SARS-CoV-2 could be present in stool samples.

Even if viral nucleotides cannot be detected in oral swabs, they can be found in anal swabs or blood ${ }^{[18]}$. Furthermore, there is a rising number of studies that have detected SARSCoV-2 RNA in stool samples. Worryingly, the constant positive detection of SARS-CoV-2 RNA from feces, indicates virions are secreted from the virus-infected gastrointestinal cells. For instance, in a China study consisting of 73 COVID-19 patients, 39 (53.42\%) patients were found to have SARS-CoV-2 RNA in stool, whereas 17 (23.29\%) patients remained positive for SARS-CoV-2 RNA in stool with a duration of positive stool ranging from 1-12 days despite negative results in respiratory samples ${ }^{[14]}$. A study from China also showed an asymptomatic 10 year old child who had persistently positive stool samples for 26 days even 
though respiratory specimens were persistently negative ${ }^{[39]}$. Taken together, these indicate that the conventional testing using RT-PCR of nasopharyngeal swabs alone may not be an accurate diagnostic test to detect the presence or clearance of SARS-CoV-2 RNA.

Importantly, a study based on patients from Wuhan also found that there could be a possible shift from oral positive during early infection to anal swab positive during late infection $^{[18]}$. SARS-CoV-2 can be detected in the intestine of COVID-19 infected patients at early or late stages, whereas SARS-CoV and MERS-CoV infected individuals, intestinal infections were detected at later stages ${ }^{[18,40-42]}$. This may be related to the cycle threshold (CT) value. A lower CT value corresponds to a higher viral load and CT value $<40$ indicates positive SARS-CoV-2 RNA. Wang et al showed there is a similar mean cycle threshold between feces (31.4), sputum (31.1) and pharyngeal (32.1) swabs of SARS-CoV-2 RNA, while nasal swab had the lowest mean cycle threshold of $24.3^{[17]}$. In the US's first COVID19 case, despite initial mild symptoms at presentation, the nasopharyngeal and oropharyngeal specimens obtained on illness day 4 showed CT values of 18-20 and 21-22 respectively. On illness day 7, the CT value of nasopharyngeal specimens increased to 23-24 while stool specimens showed greater CT value of 36-38. But, on illness day 11 and 12, there is a trend of decreasing viral levels observed for nasopharyngeal and oropharyngeal specimens ${ }^{[16]}$. Furthermore, a study found that in $70.3 \%$ of patients, prolonged shedding of SARS-CoV-2 RNA was detected in stool rather than respiratory samples, which could be up to $\geq 33$ days from illness onset ${ }^{[22]}$. Similarly, in a SARS patient, even postmortem examination (a few days after death of patients) of biopsy specimens of the small intestine yielded viable SARS$\mathrm{CoV}$ when all other organ tissues collected during autopsy had no viable virus recovered. In addition, non-viable SARS-CoV can be detected in stool specimens by PCR for up to 73 days from symptom onset ${ }^{[36]}$. Thus, it seems like that the shift from oral positive to anal positive can be seen via the trend in CT value throughout the course of illness and the prolonged shedding of SARS-CoV-2.

\section{Implications of COVID-19 on the Liver}

COVID-19 infection may have an effect on liver enzymes and other indices of hepatic function. One study hypothesized that liver tissue injury is caused by the upregulation of ACE-2 expression in liver tissue as a result of compensatory proliferation of hepatocytes derived from bile duct epithelial cells ${ }^{[43]}$. Various studies have reported various rates of prevalence of liver injury in COVID-19, ranging between $15 \%$ and $78 \%{ }^{[44]}$. In a single-center study consisting of 148 COVID-19 positive patients, 55 (37.2\%) patients had abnormal liver function upon hospital admission and this cohort were hospitalized longer ${ }^{[45]}$. Besides that, in the first reported case in the US, the patient had raised levels of creatine kinase and changes 
in hepatic function measures whereby on day 5 of hospitalization, levels of alkaline phosphatase (ALP)(68 U per liter), alanine aminotransferase (ALT)(105 U per liter), aspartate aminotransferase (AST)(77 U per liter), and lactate dehydrogenase (LDH)(465 U per liter) were all raised ${ }^{[16]}$. Another meta-analysis also showed a mild increase in ALP, ALT and AST in $4.6 \%, 20.6 \%$, and $22.8 \%$ COVID-19 patients and a mild reduction in serum bilirubin $(39.8 \%)^{[44]}$.

The implications of COVID-19 on the liver seems to follow a pattern as seen in MERS, as the concentrations of ALT, AST and LDH were elevated in five (11\%), seven $(15 \%)$ and $23(49 \%)$ MERS patients, respectively ${ }^{[37]}$. Nonetheless, findings linking liver injury with COVID-19 is still debatable. It is unsure why there are dissimilarities among different studies. For instance, Pan et al and Wu et al found COVID-19 patients had no significant liver injury ${ }^{[23,46]}$, however 2 other studies stated otherwise ${ }^{[47,48]}$. There is also study on the pathological analysis of liver tissue from a deceased COVID-19 patient that showed no viral inclusion in the liver ${ }^{[49,50]}$. Hence, more studies must be conducted to fully understand the association of COVID-19 and hepatic function abnormalities.

\section{Possible Reasons for Gastrointestinal Symptoms Following COVID-19}

There are several reasons gastrointestinal symptoms manifest quite frequently following COVID-19 infection, and they seem to be associated with the human host receptor ACE-2 ${ }^{[23]}$, the intestinal microflora ${ }^{[23]}$, the use of antibiotics and antiviral medicines ${ }^{[51,52]}$, and the direct or indirect inflammatory response as SARS-CoV-2 damages the digestive system $^{[53]}$ (Figure 1).

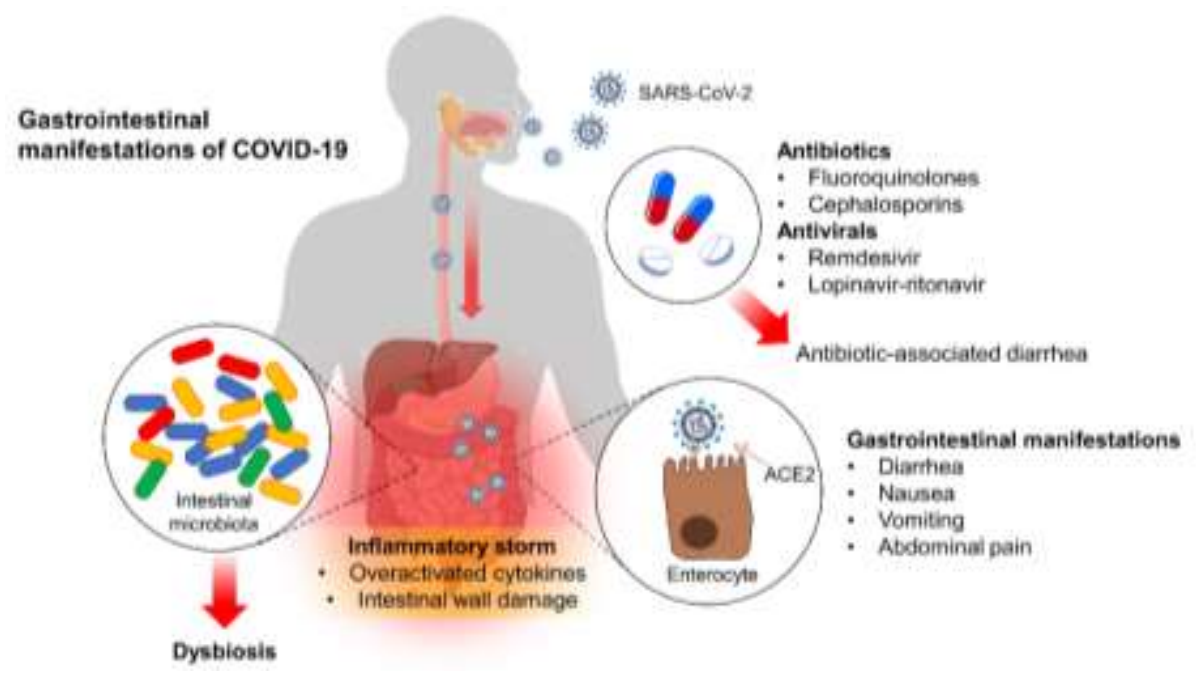

Figure 1. The possible explanation for gastrointestinal manifestations following SARS-CoV-2 infection.

Firstly, SARS-CoV-2 entry into host cells is mediated by the binding of viral surface spike protein to the host receptor ACE-2 ${ }^{[54,55]}$. ACE-2 is expressed in Type II alveolar cells 
in the lungs and goblet cells in the nasal mucosa ${ }^{[54,56]}$. However, ACE-2 is also abundantly present in the glandular cells of gastric, duodenal, and rectal epithelium. There is marked variation throughout the GI tract as ACE-2 staining is seldom found in esophageal mucosa, probably because ACE-2 is less expressed in squamous epithelial cells (esophageal epithelium) than glandular epithelial cells ${ }^{[14]}$. Another study showed ACE-2 is expressed primarily on the luminal surface of differentiated small intestinal epithelial cells, while crypt cells and the colon have lower expressions ${ }^{[57]}$. Given that the infectivity of SARS-CoV-2 is determined by the binding affinity of ACE- 2 receptors ${ }^{[22]}$, the high levels of these receptors indicate that the virus could possibly infect and replicate within the gastrointestinal tract ${ }^{[10]}$. Furthermore, intestinal inflammation is modulated by ACE-2 ${ }^{[57]}$, so it may be possible that the disruption of ACE-2 function by the SARS-CoV-2 results in diarrhea ${ }^{[22]}$. ACE-2 also has a RAS independent function, it regulates the intestinal amino acid homeostasis, is involved in the expression of antimicrobial peptides and is associated with the gut microbial ecology ${ }^{[57]}$. Amino acid malnutrition can result in intestinal inflammation and diarrhea; ACE2 mutants demonstrated reduced expression of antimicrobial peptides and an altered gut microbial composition. This leads to the speculation of an association between COVID-19 and the gut microbiota ${ }^{[57,58]}$.

Secondly, the intestinal microbiota. The human intestine houses a diverse and huge amount of gut microbiota. For instance, comparing sites in the gastrointestinal system, the colon has the most microbes, consisting of $33 \%$ of total bacteria cells in the human body ${ }^{[59]}$. The gut microbiota plays vital roles including supporting the host's metabolism, defending the host against harmful pathogens through habitat colonization and immunoregulatory responses, and regulating the development and maturation of the body's immune system ${ }^{[23}$, ${ }^{59-64]}$. Reasons for changes in gut flora include the disease itself, concomitant infections, the use of antimicrobials, and an increase in proinflammatory mediators as a result of viralinduced inflammation ${ }^{[52]}$. Alterations to the composition and function of the gastrointestinal tract microbiota may affect the respiratory tract through the common mucosal immune system, and respiratory tract flora disorders also influence the digestive tract via immune regulation. This is the gut-lung axis effect ${ }^{[23,65,66]}$, which possibly explains why digestive symptoms occur following COVID-19 infection. Hence, probiotics may be a new treatment option or an adjuvant treatment. In the treatment of severe COVID-19 infections, probiotics is recommended by the guidance (version 5) established by the China's National Health Commission and National Administration of Traditional Chinese Medicine, to maintain the balance of intestinal microecology and the prevention of secondary bacterial infection, indicating that first-line medical staffs and the Chinese government trust the importance of the role of gut microbiota in COVID-19 infection ${ }^{[58,67]}$. 
Thirdly, the use of antibiotics and antiviral drugs could result in gastrointestinal symptoms in some COVID-19 patients. Patients suspected with secondary bacterial infection are commonly treated with antibiotics in which some antibiotics (fluoroquinolones and cephalosporins) can cause antibiotic-associated diarrhea. Furthermore, antimicrobial use can have serious effects on the gut microbiota, antibody production, and immune system which could prolong the clearance of SARS-CoV-2 from the gut ${ }^{[52]}$. In patients being treated with antiviral drugs such as remdesivir, chloroquine phosphate, hydroxychloroquine, and lopinavir-ritonavir, the medications themselves can also cause diarrhea ${ }^{[51,52]}$.

Fourthly, SARS-CoV-2 can cause direct or indirect damage to the digestive system via the inflammatory response ${ }^{[9]}$. SARS-CoV-2 infection could cause an "inflammatory storm", in which overactivated cytokines, inflammatory storms, and immune dysregulation causes inflammatory damage to the intestine resulting in diarrhea ${ }^{[9,68]}$. With mounting evidence showing the presence of SARS-CoV-2 RNA in stool specimens of COVID-19 patients, it might suggest SARS-CoV-2 directly damages the intestinal mucosa, causing digestive symptoms such as diarrhea.

\section{Conclusions}

Gastrointestinal manifestations seem to be part of the course of COVID-19 infection and presentation with symptoms including - but not limited to - diarrhea, nausea and/ or vomiting and abdominal pain, should definitely not be taken lightly. Some of the possible causes of gastrointestinal manifestations seems to be associated with direct viral invasion utilizing the ACE-2 receptor ${ }^{[23]}$, alterations in the intestinal microflora ${ }^{[23]}$, the use of antibiotics and antiviral medicines ${ }^{[51,52]}$, and the direct or indirect inflammatory response as SARS-CoV-2 damaging the digestive system ${ }^{[9]}$. As COVID-19 severity increases, the digestive symptoms are more distinct. Clinicians should be alert to patients presenting with gastrointestinal symptoms due to the important implications as this cohort of patients have much longer duration from symptom onset to admission resulting in late diagnosis and treatment, compared to patients without digestive symptoms. It is possible that the increased viral load and replication in the gastrointestinal tract (GIT) results in more severe disease and the delay in reporting by patients with extrapulmonary symptoms but without the typical respiratory symptoms led to a later and less curable stage ${ }^{[23]}$. This is consistent with a metaanalysis that showed a higher prevalence of gastrointestinal symptoms in those with severe COVID-19 than non-severe COVID-19 (17.1\% vs $11.8 \%)^{[22]}$. Importantly, with the detection of SARS-CoV-2 in specimens from other sites other than the nasopharyngeal site, it is likely that SARS-CoV-2 can be transmitted in ways other than respiratory droplets. It is also likely that the virus can infect and replicate in other sites, in this case, the gastrointestinal tract of the human host. This suggests possible tissue tropism of SARS-CoV-2 in the intestinal cells and a possible fecal-oral transmission. 
With these findings, there will be implications on the disease transmission, viral detection, infection control and management. Hence, changes must be made to efficiently combat the worsening of SARS-CoV-2. Clinicians and health care workers should be more cautious if patients present with initial gastrointestinal symptoms and take precautionary measures including droplet and fomite precautions, proper handling of COVID-19 patients' excreta, and have barrier precautions when performing colonoscopy ${ }^{[36]}$. Furthermore, testing of stool specimens for SARS-CoV-2 RNA should be made in addition to respiratory specimens. Efforts should also be made to alert all people on the gastrointestinal manifestations of COVID-19 so there can be early detection, diagnosis, isolation, and intervention, and reduce SARS-CoV-2 transmission within the family and in the community.

Author Contributions: AY-KT performed the literature search, critical data analysis as well as manuscript writing. PP, LT-HT, JW-FL, VL, and L-HL provided review, editing, and proofreading for the manuscript. VL and L-HL conceptualize the project.

Funding: The SEED Funding funded this work from Microbiome and Bioresource Research Strength (MBRS), Jeffrey Cheah School of Medicine and Health Sciences, (Vote Number: MBRS/JCSMHS/02/2020) awarded to VL.

Acknowledgments: Professor Dr. Shajahan Yasin, Professor, and Head of School, Jeffrey Cheah School of Medicine and Health Sciences, Monash University Malaysia.

Conflicts of Interest: The authors declare no conflict of interest.

\section{References}

1. Johnson D, Ren SEC, Johnson HD, et al. COVID-19: Are Malaysians embracing or suffering the new normality? Prog Microbes Mol Biol 2020; 3(1): a0000102.

2. World Health Organization. WHO announces COVID-19 outbreak a pandemic. 2020 [Accessed 10 November 2021]; Available from: https://www.euro.who.int/en/health-topics/healthemergencies/coronavirus-covid-19/news/news/2020/3/who-announces-covid-19-outbreak-apandemic.

3. Thye AY-K, Loo K-Y, Tan KBC, et al. Insights into COVID-19 Delta variant (B. 1.617. 2). Prog Microbes Mol Biol 2021; 4(1): a0000243.

4. Letchumanan V, Ab Mutalib N-S, Goh B-H, et al. Novel coronavirus 2019-nCoV: Could this virus become a possible global pandemic. Prog Microbes Mol Biol 2020; 3(1): a0000068.

5. Loo K-Y, Letchumanan V, Ser H-L, et al. COVID-19: Insights into potential vaccines. Microorganisms 2021; 9(3): 605.

6. Tan LT-H, Letchumanan V, Ser H-L, et al. PMMB COVID-19 Bulletin: United Kingdom (22nd April 2020). Prog Microbes Mol Biol 2020; 3(1): a0000078.

7. Ser H-L, Letchumanan V, Law JW-F, et al. PMMB COVID-19 Bulletin: Spain (18th April 2020). Prog Microbes Mol Biol 2020; 3(1): a0000074.

8. Lee VS, Chong WL, Sukumaran SD, et al. Computational screening and identifying binding interaction of anti-viral and anti-malarial drugs: Toward the potential cure for SARS-CoV-2. Prog Drug Discov Biomed Sci 2020; 3(1): a0000065. 
9. $\mathrm{Wu} \mathrm{Y}$, Ho W, Huang $\mathrm{Y}$, et al. SARS-CoV-2 is an appropriate name for the new coronavirus. Lancet 2020; 395(10228): 949-950.

10. Wong SH, Lui RN, and Sung JJ. Covid- 19 and the digestive system. J Gastroenterol Hepatol 2020; 35(5): 744-748.

11. Lu R, Zhao X, Li J, et al. Genomic characterisation and epidemiology of 2019 novel coronavirus: implications for virus origins and receptor binding. Lancet 2020; 395(10224): 565-574.

12. Johns Hopkins Coronavirus Resource Center. COVID-19 Dashboard by the Center for Systems Science and Engineering (CSSE) at Johns Hopkins University (JHU). 2020 [Accessed 10 November 2021]; Available from: https://coronavirus.jhu.edu/map.html.

13. Loo K-Y and Letchumanan V. COVID-19: Malaysia's fight against this deadly virus. Prog Microbes Mol Biol 2021; 4(1): a0000204.

14. Xiao $\mathrm{F}$, Tang $\mathrm{M}$, Zheng $\mathrm{X}$, et al. Evidence for gastrointestinal infection of SARS-CoV-2. Gastroenterology 2020; 158(6): 1831-1833. e3.

15. Gu J, Han B, and Wang J. COVID-19: gastrointestinal manifestations and potential fecal-oral transmission. Gastroenterology 2020; 158(6): 1518-1519.

16. Holshue ML, DeBolt C, Lindquist S, et al. First case of 2019 novel coronavirus in the United States. N Engl J Med 2020; 382: 929-936.

17. Wang W, Xu Y, Gao R, et al. Detection of SARS-CoV-2 in different types of clinical specimens. JAMA 2020; 323(18): 1843-1844.

18. Zhang W, Du R-H, Li B, et al. Molecular and serological investigation of 2019-nCoV infected patients: implication of multiple shedding routes. Emerg Microbes Infect 2020; 9(1): 386-389.

19. Xu CL, Raval M, Schnall JA, et al. Duration of respiratory and gastrointestinal viral shedding in children with SARS-CoV-2: a systematic review and synthesis of data. J Pediatr Infect Dis 2020; 39(9): e249-e256.

20. Xu Y, Li X, Zhu B, et al. Characteristics of pediatric SARS-CoV-2 infection and potential evidence for persistent fecal viral shedding. Nat Med 2020; 26(4): 502-505.

21. Harmer D, Gilbert M, Borman R, et al. Quantitative mRNA expression profiling of ACE 2, a novel homologue of angiotensin converting enzyme. FEBS Lett 2002; 532(1-2): 107-110.

22. Cheung KS, Hung IF, Chan PP, et al. Gastrointestinal manifestations of SARS-CoV-2 infection and virus load in fecal samples from a Hong Kong cohort: systematic review and meta-analysis. Gastroenterology 2020; 159(1): 81-95.

23. Pan L, Mu M, Yang P, et al. Clinical characteristics of COVID-19 patients with digestive symptoms in Hubei, China: a descriptive, cross-sectional, multicenter study. Am J Gastroenterol 2020; 115(5): 766-773.

24. Chen N, Zhou M, Dong X, et al. Epidemiological and clinical characteristics of 99 cases of 2019 novel coronavirus pneumonia in Wuhan, China: a descriptive study. Lancet 2020; 395(10223): 507-513.

25. Huang C, Wang Y, Li X, et al. Clinical features of patients infected with 2019 novel coronavirus in Wuhan, China. Lancet 2020; 395(10223): 497-506.

26. Liu K, Fang Y-Y, Deng Y, et al. Clinical characteristics of novel coronavirus cases in tertiary hospitals in Hubei Province. Chin Med J 2020; 133(9): 1025.

27. Lu X, Zhang L, Du H, et al. SARS-CoV-2 infection in children. N Engl J Med 2020; 382(17): 16631665. 
28. Zhou F, Yu T, Du R, et al. Clinical course and risk factors for mortality of adult inpatients with COVID-19 in Wuhan, China: a retrospective cohort study. Lancet 2020; 395(10229): 1054-1062.

29. Shi H, Han X, Jiang N, et al. Radiological findings from 81 patients with COVID-19 pneumonia in Wuhan, China: a descriptive study. Lancet Infect Dis 2020; 20(4): 425-434.

30. Wang D, Hu B, Hu C, et al. Clinical characteristics of 138 hospitalized patients with 2019 novel coronavirus-infected pneumonia in Wuhan, China. JAMA 2020; 323(11): 1061-1069.

31. Yang X, Yu Y, Xu J, et al. Clinical course and outcomes of critically ill patients with SARS-CoV-2 pneumonia in Wuhan, China: a single-centered, retrospective, observational study. Lancet Respir Med 2020; 8(5): 475-481.

32. $\mathrm{Xu} \mathrm{X}-\mathrm{W}, \mathrm{Wu} \mathrm{X}-\mathrm{X}$, Jiang X-G, et al. Clinical findings in a group of patients infected with the 2019 novel coronavirus (SARS-Cov-2) outside of Wuhan, China: retrospective case series. BMJ 2020; 368 : m606.

33. Mantovani A, Rinaldi E, Zusi C, et al. Coronavirus disease 2019 (COVID-19) in children and/or adolescents: a meta-analysis. Pediatr Res 2021; 89(4): 733-737.

34. Castagnoli R, Votto M, Licari A, et al. Severe acute respiratory syndrome coronavirus 2 (SARS-CoV2) infection in children and adolescents: a systematic review. JAMA Pediatr 2020; 174(9): 882-889.

35. Akobeng AK, Grafton-Clarke C, Abdelgadir I, et al. Gastrointestinal manifestations of COVID-19 in children: a systematic review and meta-analysis. Frontline Gastroenterol 2021; 12(4): 332-337.

36. Leung WK, To K-f, Chan PK, et al. Enteric involvement of severe acute respiratory syndromeassociated coronavirus infection. Gastroenterology 2003; 125(4): 1011-1017.

37. Assiri A, Al-Tawfiq JA, Al-Rabeeah AA, et al. Epidemiological, demographic, and clinical characteristics of 47 cases of Middle East respiratory syndrome coronavirus disease from Saudi Arabia: a descriptive study. Lancet Infect Dis 2013; 13(9): 752-761.

38. Choi WS, Kang C-I, Kim Y, et al. Clinical presentation and outcomes of Middle East respiratory syndrome in the Republic of Korea. Infect Chemother 2016; 48(2): 118-126.

39. Tang A, Tong Z-d, Wang H-l, et al. Detection of novel coronavirus by RT-PCR in stool specimen from asymptomatic child, China. Emerg Infect Dis 2020; 26(6): 1337.

40. Shi X, Gong E, Gao D, et al. Severe acute respiratory syndrome associated coronavirus is detected in intestinal tissues of fatal cases. Am J Gastroenterol 2005; 100(1): 169-176.

41. Ding Y, He L, Zhang Q, et al. Organ distribution of severe acute respiratory syndrome (SARS) associated coronavirus (SARS- CoV) in SARS patients: implications for pathogenesis and virus transmission pathways. J Pathol 2004; 203(2): 622-630.

42. Zhou J, Li C, Zhao G, et al. Human intestinal tract serves as an alternative infection route for Middle East respiratory syndrome coronavirus. Sci Adv 2017; 3(11): eaao4966.

43. Guan G-W, Gao L, Wang J-W, et al. Exploring the mechanism of liver enzyme abnormalities in patients with novel coronavirus-infected pneumonia. Chin J Hepatol 2020; 28(2): 100-106.

44. Zarifian A, Zamiri Bidary M, Arekhi S, et al. Gastrointestinal and hepatic abnormalities in patients with confirmed COVID- 19: A systematic review and meta- analysis. J Med Virol 2021; 93(1): 336350 .

45. Fan Z, Chen L, Li J, et al. Clinical features of COVID-19-related liver functional abnormality. Clin Gastroenterol Hepatol 2020; 18(7): 1561-1566. 
46. Wu J, Liu J, Zhao X, et al. Clinical characteristics of imported cases of coronavirus disease 2019 (COVID-19) in Jiangsu Province: a multicenter descriptive study. Clin Infect Dis 2020; 71(15): 706712.

47. Yao N, Wang S, Lian J, et al. Clinical characteristics and influencing factors of patients with novel coronavirus pneumonia combined with liver injury in Shaanxi region. Chin J Hepatol 2020; 28: E003E003.

48. Hu L-L, Wang W-J, Zhu Q-J, et al. Novel coronavirus pneumonia-related liver injury: etiological analysis and treatment strategy. Chin J Hepatol 2020; 28(2): 97-99.

49. Lotfi M and Rezaei N. SARS- CoV- 2: A comprehensive review from pathogenicity of the virus to clinical consequences. J Med Virol 2020; 92(10): 1864-1874.

50. Xu Z, Shi L, Wang Y, et al. Pathological findings of COVID-19 associated with acute respiratory distress syndrome. Lancet Respir Med 2020; 8(4): 420-422.

51. Ye Q, Wang B, Zhang T, et al. The mechanism and treatment of gastrointestinal symptoms in patients with COVID-19. Am J Physiol Gastrointest Liver Physiol 2020; 319(2): G245-G252.

52. Perisetti A, Gajendran M, and Goyal H. Putative mechanisms of diarrhea in COVID-19. Clin Gastroenterol Hepatol 2020; 18(13): 3054-3055.

53. Wang F, Zheng S, Zheng C, et al. Attaching clinical significance to COVID-19-associated diarrhea. Life Sci 2020; 260: 118312.

54. Thye AY-K, Law JW-F, Pusparajah P, et al. Emerging SARS-CoV-2 Variants of Concern (VOCs): An Impending Global Crisis. Biomedicines 2021; 9(10): 1303.

55. Shang J, Ye G, Shi K, et al. Structural basis of receptor recognition by SARS-CoV-2. Nature 2020; 581(7807): 221-224.

56. Hikmet F, Méar L, Edvinsson Å, et al. The protein expression profile of ACE2 in human tissues. Mol Syst Biol 2020; 16(7): e9610.

57. Hashimoto T, Perlot T, Rehman A, et al. ACE2 links amino acid malnutrition to microbial ecology and intestinal inflammation. Nature 2012; 487(7408): 477-481.

58. Gao QY, Chen YX, and Fang JY. 2019 Novel coronavirus infection and gastrointestinal tract. J Dig Dis 2020; 21(3): 125.

59. Lee L-H, Wong SH, Chin S-F, et al. Human Microbiome: Symbiosis to Pathogenesis. Front Microbiol 2021; 12: Article 605783.

60. Iacob S, Iacob DG, and Luminos LM. Intestinal microbiota as a host defense mechanism to infectious threats. Front Microbiol 2019; 9: 3328.

61. Laville E, Perrier J, Bejar N, et al. Investigating host microbiota relationships through functional metagenomics. Front Microbiol 2019; 10: 1286.

62. Rodionov DA, Arzamasov AA, Khoroshkin MS, et al. Micronutrient requirements and sharing capabilities of the human gut microbiome. Front Microbiol 2019; 10: 1316.

63. Sharma V, Rodionov DA, Leyn SA, et al. B-vitamin sharing promotes stability of gut microbial communities. Front Microbiol 2019; 10: 1485.

64. Shin J, Noh J-R, Chang D-H, et al. Elucidation of Akkermansia muciniphila probiotic traits driven by mucin depletion. Front Microbiol 2019; 10: 1137.

65. Budden KF, Gellatly SL, Wood DL, et al. Emerging pathogenic links between microbiota and the gutlung axis. Nat Rev Microbiol 2017; 15(1): 55-63. 
66. He Y, Wen Q, Yao F, et al. Gut-lung axis: the microbial contributions and clinical implications. Crit Rev Microbiol 2017; 43(1): 81-95.

67. National Health Committee of the People's Republic of China National Administration of Traditional Chinese Medicine. Diagnostic and therapeutic guidance for 2019 novel coronavirus disease (version 5). 2020 [Accessed 10 November 2021]; Available from: http://www.nhc.gov.cn/yzygj/s7653p/202002/d4b895337e19445f8d728fcaf1e3e13a/files/ab6bec7f93 e64e7f998d802991203cd6.pdf.

68. Liu J, Li S, Liu J, et al. Longitudinal characteristics of lymphocyte responses and cytokine profiles in the peripheral blood of SARS-CoV-2 infected patients. EBioMedicine 2020; 55: 102763.

Author(s) shall retain the copyright of their work and grant the Journal/Publisher right for the first publication with the work simultaneously licensed under:

Creative Commons Attribution-NonCommercial 4.0 International (CC BY-NC 4.0). This license allows for the copying, distribution and transmission of the work, provided the correct attribution of the original creator is stated. Adaptation and remixing are also permitted. 